\title{
REDUCCIÓN DEL MANEJO DE MATERIALES EN LÍNEA EN UNA ENSAMBLADORA DE AUTOS MEDIANTE LA APLICACIÓN DE LEAN MANUFACTURING
}

\author{
ANTONIO HUERTA ESTÉVEZ* \\ https://orcid.org/0000-0003-3600-4540 \\ Tecnológico Nacional de México / Instituto Tecnológico de Veracruz
}

Recibido: 10 de octubre del 2020 / Aprobado: 28 de noviembre del 2020

doi: https://doi.org/10.26439/ing.ind2021.n40.4880

RESUMEN: Este artículo se enfoca en la aplicación de lean manufacturing a una planta ensambladora de autos, la cual contaba con paros de línea y largos recorridos que generaban pérdidas económicas causadas por incumplimiento en la entrega de unidades a tiempo por parte de los distribuidores. Al final, se redujeron los paros de línea hasta el 50 \% y una cantidad de 383751 dólares en el inventario.

PALABRAS CLAVE: manufactura esbelta / paros de línea / costos de operación / manejo de materiales / herramientas lean

REDUCTION OF MATERIAL HANDLING IN AN AUTOMOBILE ASSEMBLY LINE THROUGH LEAN MANUFACTURING

ABSTRACT: This article focuses on the application of lean manufacturing principles to an automobile assembly plant, which had production line stoppages and long runs that generated losses caused by failure to deliver the units on time to dealers. In the end, line stoppages were reduced by up to $50 \%$ that generated savings of $\$ 383,751$.

KEYWORDS: lean manufacturing / line stoppages / operating costs / material handling / lean tools

\footnotetext{
*Correo electrónico: huertastvz@gmail.com
} 


\section{INTRODUCCIÓN}

Contar con una correcta administración de operaciones y suministros que desempeñe el trabajo en forma expedita, eficiente, sin errores y a bajo costo es vital en los sistemas de producción (Chase, Jacobs y Aquilano, 2009). Por ello las organizaciones implementan diferentes herramientas para incrementar su eficiencia y eficacia; de esta manera ser elegidos por el consumidor, el cual requiere menores precios y tiempos de respuesta sin decremento de la calidad (Vargas-Hernández, Jiménez Castillo y Muratalla-Bautista, 2018). La manufactura esbelta (lean manufacturing) establece cómo mejorar un sistema de producción concentrándose en la eliminación de los desperdicios, los cuales pueden definirse como: aquellas actividades que utilizan más recursos de los mínimos necesarios (esperas, sobreproducción, exceso de inventarios, defectos, transporte, movimientos y retrabajos) (Figueredo Lugo, 2015).

Herramientas de manufactura esbelta como el sistema Kanban surgió para satisfacer la necesidad de las organizaciones tecnológicas para responder a cambios frecuentes en su demanda, tal como el caso de la industria automotriz, además de facilitar el trabajo en equipo (Anderson y Bozheva, 2019). De igual manera, herramientas como los mapas de valor y el diagrama de espagueti, facilitaron el reconocimiento de procesos y distancias a mejorar para la disminución en los tiempos de espera, como también la identificación de desperdicios, eliminando así los procesos responsables de dichos desperdicios (Martínez Sánchez, Martínez Flores, Nuño de la Parra y Cavazos Arroyo, 2016).

Se ha llevado a cabo con gran éxito el uso de manufactura flexible, la cual reduce tiempos de preparación y mejora tanto el flujo de materiales e inventario en procesos, como la administración de la calidad del producto y de la eficiencia en producción (Bravo, Orejuela y Menduiña, 2012). El uso de herramientas de lean manufacturing hacen disminuir del 1,6 \% a $0 \%$ las mermas por falta de suministros lo que impacta en un ahorro de 48450 dólares en el costo de espera de los materiales (Franco, Jiménez García y Ruelas Santoyo, 2019). Las implementaciones de algunas herramientas lean dan como resultado la reducción del 32,73 \% del tiempo de ciclo, adicionalmente, el tiempo de producción baja en un $11,8 \%$ y la productividad aumenta en un 16,66 \% (Lijalem, 2020).

\section{DESARROLLO DEL MÉTODO}

La investigación es de tipo aplicada, porque se buscaba resolver uno o varios problemas usando conocimientos ya probados, técnicas y métodos de propósitos específicos. Como primer paso, se determinó el estado en el cual se encontraba el sistema, en el cual se encontró que los paros de la línea de producción al mes estaban en promedio en los 370 minutos, además de 1600 metros por cada ciclo de recorrido de los carros secuenciadores que alimentan de materiales a la línea de ensamble, esto generaba altos costos de 
operación en el área de manejo de materiales provocando pérdidas económicas significativas por los incumplimientos en las entregas de las unidades a los distribuidores.

En base a lo mencionado, previamente, se fijó como siguiente medida el designar un proveedor externo (outsourcing) para el secuenciador de los materiales a proveer a la línea de producción. De acuerdo con Rodenes Adam, Moncaleano Rodríguez y Martínez Aparisi (2009), la gestión del suministro para las empresas: logística y distribución de bienes, almacenamiento y gestión de inventarios, diseño y fabricación de servicios y productos, y gestión de compras, por parte de un outsourcing, representa el $36 \%$ del mercado que abarcan estos (Rodenes Adam et al., 2009).

Posteriormente, se le destinó un lugar, dentro de las instalaciones de la planta, para que se ubicara este proveedor, convirtiéndose en el encargado del secuenciado de partes, significando esto una gran ventaja, ya que al estar dentro de las instalaciones de la ensambladora, se reducirían los recorridos de las secuencias evitando daños a los materiales, y así habría menos paros de línea, objetivo principal de este trabajo.

Hoy en día, los sistemas de producción deben ser adaptables para dar una respuesta efectiva a las necesidades del cliente en la elaboración de nuevos productos o para satisfacer los requisitos del cliente. La rápida obsolescencia de los productos y las fluctuaciones repentinas e inesperadas de la demanda, han llevado al desarrollo de herramientas en los sistemas de producción como celdas de manufactura, la cual se basa en los principios de grupos tecnológicos, que busca agrupar productos para ser fabricados en características similares (tamaño, forma o procesamiento común) (Rodríguez León, Quiroga Méndez y Ortiz Pimiento, 2013).

Respecto a la tabla 1, en donde las molduras de puerta, que cuentan con 44 números distintos de partes, serán las de mayor complejidad para secuenciar, y las capotas, con solo dos números de parte, serán las de menor complejidad para poder secuenciarse.

Tabla 1

Partes o commodities afectadas para secuencias

\begin{tabular}{lccccc}
\hline \multicolumn{1}{c}{ Material } & Modelo & $\begin{array}{c}\text { Número } \\
\text { de parte }\end{array}$ & $\begin{array}{c}\text { Paros de } \\
\text { línea }\end{array}$ & $\begin{array}{c}\text { Paros en } \\
\text { minutos }\end{array}$ & $\begin{array}{c}\text { Puede correr } \\
\text { faltante }\end{array}$ \\
\hline Capotas & FF27 & 2 & $X$ & 12 \\
Cinturones de seguridad & JC44-FF27 & 40 & $X$ & 120 \\
Molduras de puertas & JC44-FF27 & 44 & & \\
Espejos & JC44-FF27 & 17 & & \\
Módulos NGC (computadora) & JC44-FF27 & 5 & $X$ & 20 \\
Amortiguadores delanteros & JC44-FF27 & 21 & $X$ & 10 & \\
Vistas de cargo & FF27 & 8 & & & $X$ \\
\hline
\end{tabular}

(continúa) 
(continuación)

\begin{tabular}{llcll}
\hline Vistas pilar "a" & JC44-FF27 & 8 & & \\
Molduras header & FF27 & 27 & & X \\
Amortiguadores traseros & JC44-FF27 & 7 & $X$ & 14 \\
Tubos de escape & JC44-FF27 & 9 & $X$ & 27 \\
Radiadores & JC44-FF27 & 7 & $X$ & 21 \\
Convertidor catalítico & JC44-FF27 & 7 & $X$ & 28 \\
Volantes & JC44-FF27 & 12 & $X$ & 60 \\
Filtros de aire & JC44-FF27 & 7 & $X$ & 28 \\
Rines de 17" & JC44-FF27 & 6 & $X$ & 36 \\
Cartones de puertas & JC44-FF27 & 36 & & $X$ \\
Viseras & FF27 & 4 & & $X$ \\
Vistas de espejos & JC44-FF27 & 12 & & \\
Vistas de quinta puerta & JC44-FF27 & 2 & & \\
\hline
\end{tabular}

Elaboración propia

El aumento en el grado de compromiso de los obreros se alcanzará cuando se comprenda que los individuos también son parte de la organización, esto incrementaría el sentido de pertenencia, ya que ellos mostrarían mayor compromiso hacia la organización por iniciativa propia debido al franco deseo de lograr los objetivos de la empresa (Oropesa-Vento, García-Alcaraz, Rivera y Manotas, 2015).

Por ello, en la siguiente etapa del proyecto, se procedió a revisar la estructura organizacional que tenía el proveedor en el área de secuenciados, con 50 empleados (42 sindicalizados) para cubrir la operación, y se presentaron planes de mejora a través de un programa de capacitación al personal incluyendo una matriz de habilidades, gráfica de ausentismo, planes de contingencia y rotación de personal. A continuación, se enlistan las dos principales actividades desarrolladas en esta etapa:

a) Cada operador debe desarrollar diversas habilidades en el proceso de secuenciación: con esto se asegura que cada operador (secuenciador) debe conocer por lo menos el proceso de dos o más secuenciados, teniendo las habilidades necesarias para desarrollar las actividades en varios procesos.

b) Eliminar errores en las secuencias por ausentismo o rotación: el personal deberá de capacitarse en diversas secuencias en turnos que no correspondan a su trabajo diario, así, se asegurará que, al momento de ausentarse un trabajador en un turno determinado, se pueda recurrir a otro ya capacitado en dicho turno con esa secuencia, con el debido pago respectivo por el desarrollo de esta actividad, es decir, se supliría al operador faltante y el pago se le otorga a quien labore ese turno. 
Ya establecidos los espacios para el outsourcing y generadas las acciones con la estructura organizacional de este, la siguiente etapa fue analizar el volumen anual, es decir, de las veinte familias de partes cuál sería el porcentaje secuenciado a línea, así como determinar el modelo de vehículo en que se ensamblarían, cuántas se llevarían por carro y cuál sería el número total para cada familia. La situación contemplaba 3 tráileres equipados con cajas de 53 pies, para el transporte de los carros secuenciadores del área de embarque, del secuenciador hacia las rampas de recibo de la ensambladora, en donde se descargarían, y los ruteros asignados los dirigen hasta la línea de ensamble con 3 rutas de secuenciado: JIT 1, JIT 2 y JIT 3. La figura 1 muestra el transporte de cada tráiler el cual va en dirección frontal del mismo.

\section{JIT 1}

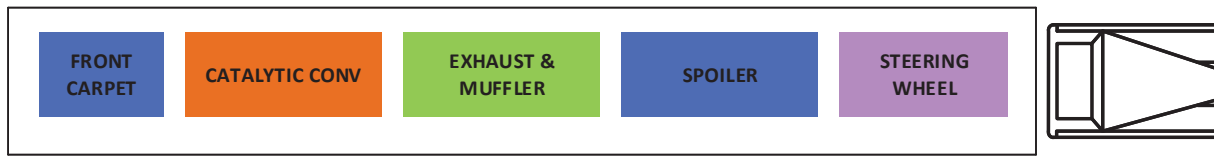

\section{JIT 2}

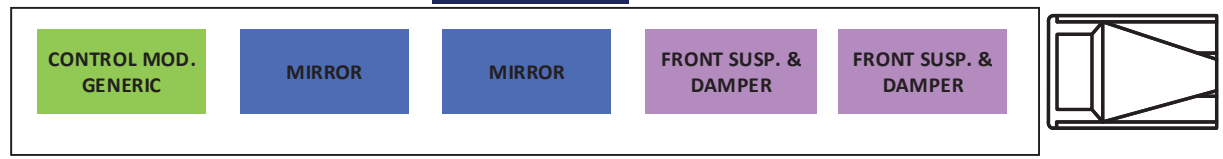

\section{JIT 3}

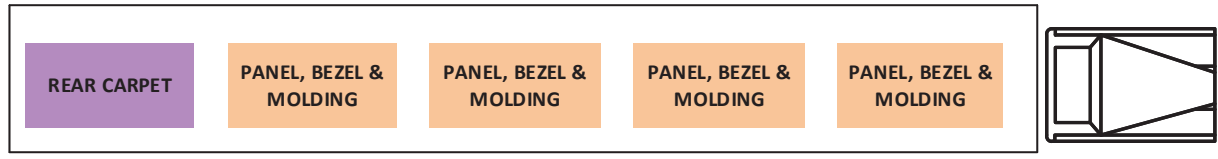

Figura 1. Equipo móvil que tenía la ensambladora

\section{Elaboración propia}

Como se ha mencionado, el modelo que estaba implementado era el uso de los 3 tráileres que entregaban 3 rutas de secuenciado justo a tiempo (JIT 1, JIT 2, JIT 3), 15 minutos para descarga y carga de las partes secuenciadas y de los contenedores, además de 5 a 10 minutos de traslado, desde el almacén del proveedor hasta la línea de ensamble, en un total de 1600 metros de recorrido en cada ciclo, lo cual implicaba un costo de 200 USD por día por tractor. 


\section{RESULTADOS}

El impacto más significativo fue el referido a la mano de obra, con un subtotal de 564000 dólares; seguido del inventario, con el cual se obtuvo un ahorro hasta de 383751 dólares; por otro lado, al no contar con partes en espera en la línea de producción, se obtuvo una reducción hasta del $50 \%$ de recorrido del almacén a la línea de producción, de 1600 a solo 800 metros, además de reducir los ciclos de 110 a solo 20 , significando una disminución del $85 \%$. Con esto se evitan daños en el manejo de las partes al tenerlas secuenciadas, lo cual impacta hasta en un ahorro de 36622 dólares anuales $(0,25$ dólares anuales por las 146488 unidades fabricadas).

Del lado izquierdo de la figura 2, se observa la caja de tráiler de 53 pies de arrastre, en donde contiene secuencias que, posteriormente, son llevadas a la línea de producción por un tractor, sin que aun sean requeridas, utilizando el sistema push. Ahora, del lado derecho de la figura, se presenta el mismo tractor una vez que enganchó el carro de secuencias y listo para ser llevado a su ruta, una vez que los solicite la línea de producción, mediante la implementación de sistemas pull, lo que representa mayor capacidad y maniobrabilidad, dejando las partes listas en secuencias a utilizar acorde a la cantidad requerida por parte de producción.
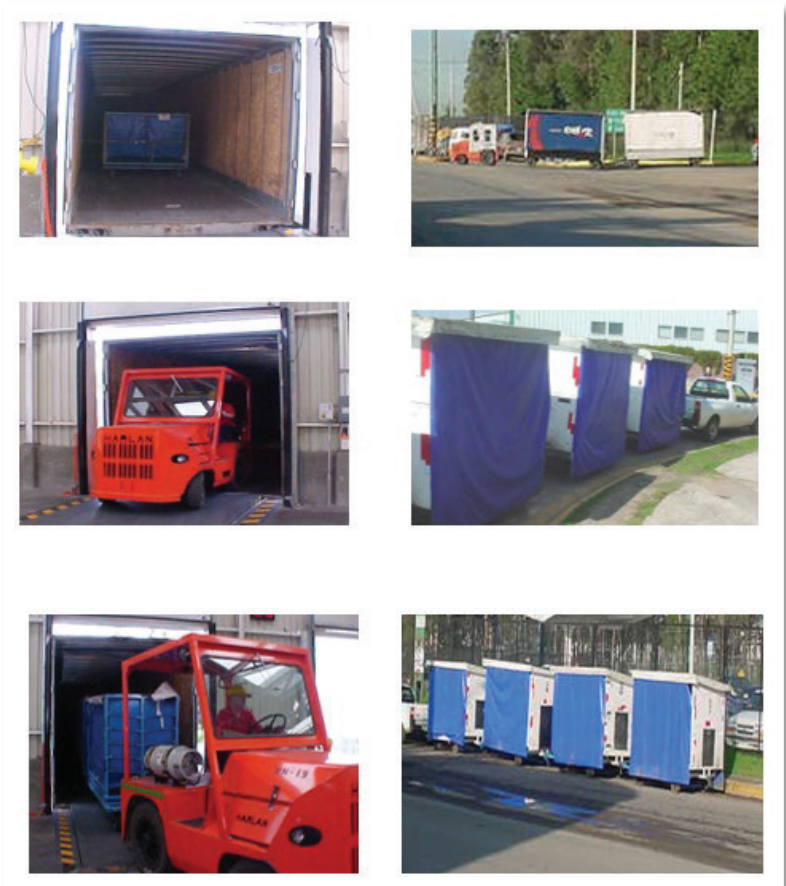

Figura 2. Situación de la ensambladora vs. situación propuesta

Elaboración propia 
Bajo este contexto, de acuerdo con Acevedo Suárez, Urquiaga Rodríguez y Gómez Acosta (2001), se puede definir la herramienta Kanban como la destreza del manejo de producción fundamentado en un sistema pull (jalar) basado en la autogestión de procesos, prescindiendo de la programación centralizada. Se produce y transporta lo que se solicita en los procesos consumidores, manteniendo en movimiento, únicamente, lo necesario que garantice la continuidad del consumo. Cuando se entorpece el consumo, se detiene la producción. Es una herramienta para conseguir la producción justo a tiempo (JIT, por sus siglas en inglés) (Chase, Jacobs y Aquilano, 2009).

La figura 3 muestra la manera en que se solicitan, mediante Kanban electrónico, las partes de las familias. En primer lugar, se genera un requerimiento en el sistema que es enviado al proveedor, de manera electrónica, el cual prepara y carga la cantidad de secuencias solicitadas en los carros que serán jalados por el tractor y llevados a la línea de producción directamente, regresando con carros vacíos, los cuales regresan al proveedor para su reabastecimiento en el momento que vuelvan a ser solicitados.
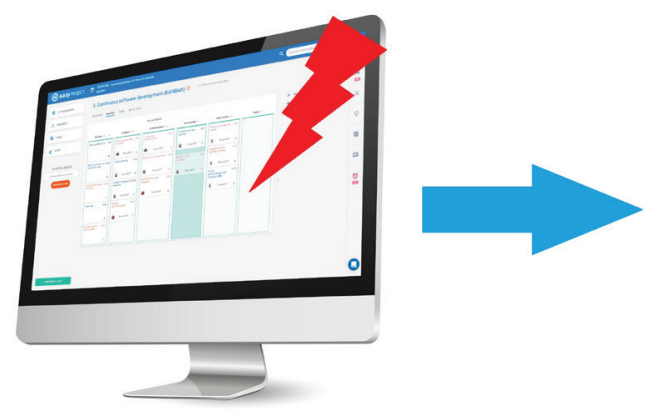

\section{PROVEEDOR}
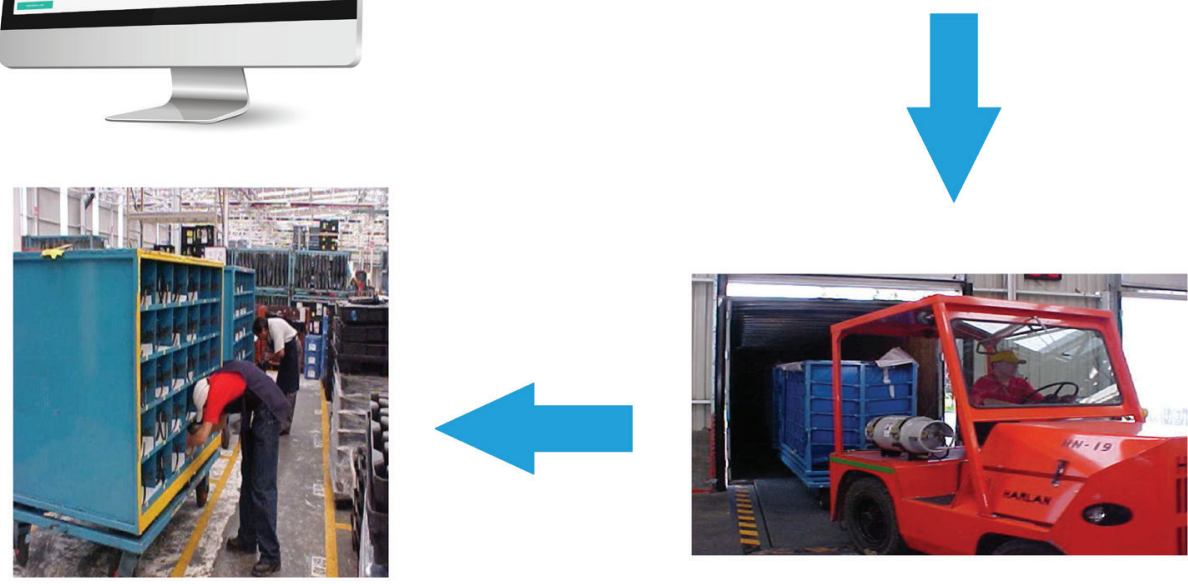

Figura 3. Secuencias de productos

\section{Elaboración propia}

Se implementaron los sistemas de producción de jalar (o pull) en donde este tipo de manufactura esbelta logra la utilización mínima de inventarios y las piezas llegan a la 
siguiente estación de trabajo justo a tiempo, bajo la lógica que nada se produce hasta que se necesita (Chase, Jacobs y Aquilano, 2009). Este método de trabajo hace más eficiente el suministro de los materiales, ya que, al tener un abastecimiento eficiente de materiales, se garantiza obtener un costo menor, debido al desperdicio representado por el tiempo de espera por falta de materiales.

En un sistema de producción, los elementos a fabricar se distribuyen desde un esquema superior hacia uno inferior. El sistema Kanban mediante una tarjeta da inicio a la tarea, en dicha tarjeta está la información que describe el material, su identificación (nombre y tiempos de producción y traslado), así como la secuencia que deberá de seguir, etc. Cada tarjeta contiene el estado de cada etapa (pendiente, en trabajo, terminada), las cuales son compartidas por todo el equipo de trabajo y tienden a minimizar la burocracia dentro del equipo (Arango Serna, Campuzano Zapata y Zapata Cortes, 2015).

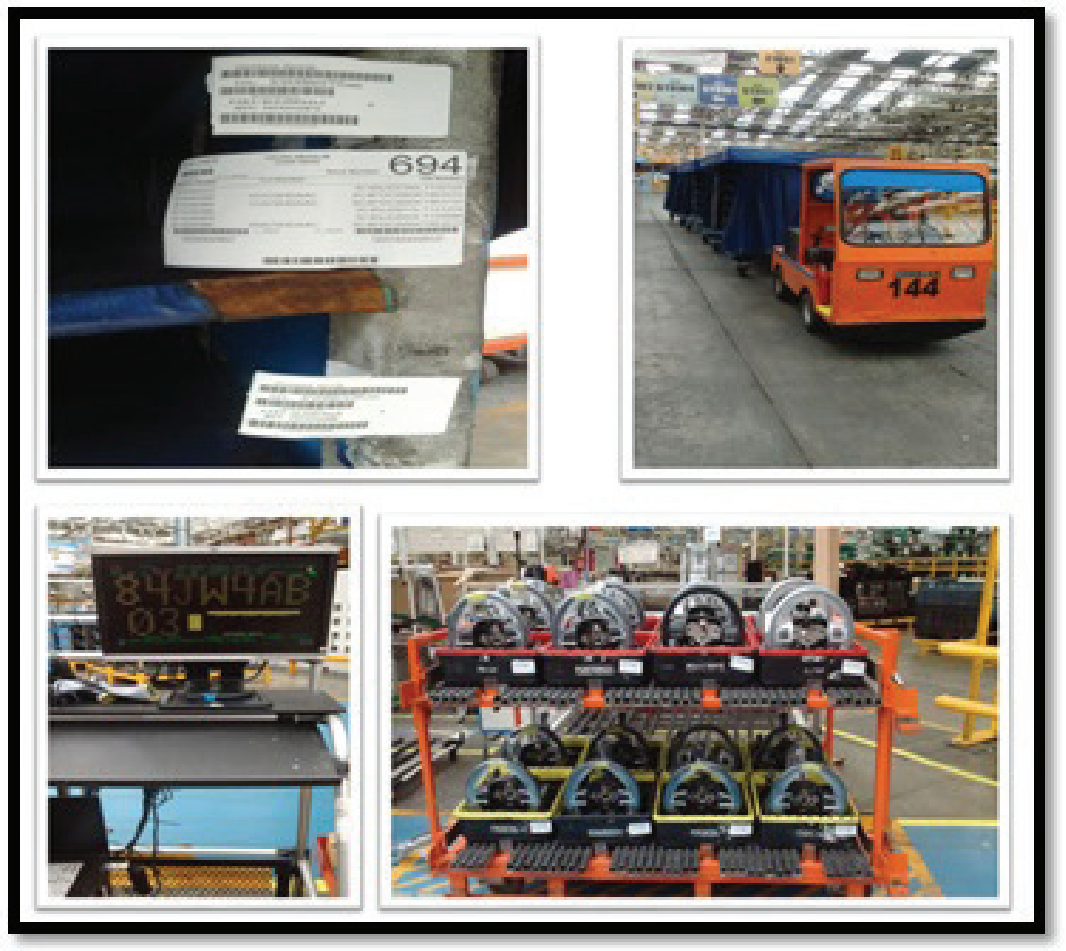

Figura 4. Aplicación del sistema Kanban

\section{Elaboración propia}

En la figura 4 se puede apreciar el uso del sistema Kanban, una de las principales herramientas base para este tipo de sistemas de manufactura esbelta, el cual apoya a la línea de ensamble mediante el uso de las tarjetas en el suministro eficiente de las 
secuencias. La idea de la manufactura esbelta es crear sistemas de producción ajustada, libre de residuos como transporte, materiales, espacios, personal y tiempo (JiménezGarcía, Mendiola-García, Medina-Flores, Mezura-Montes y Vázquez-López, 2016).

La línea ya optimizada cuenta con siete números de partes donde los materiales están perfectamente distribuidos a lo largo de la estación de trabajo y cumple con los requerimientos de producción; además, está familiarizada con la ergonomía laboral, lo que permite que el operario de producción reduzca sus caminatas y no presente problemas para abastecerse de sus materiales y así poder ensamblarlos en las unidades.

Una secuencia consiste en preparar en un carro una o varias familias de materiales, en un orden determinado, para ser enviados desde el almacén a la línea productiva que lo solicita, a esto se le conoce como "ciclo de secuencia". La figura 5 muestra como un operador recibe las partes de un secuenciador externo exactamente en su punto de instalación; de no hacerlo de esta manera, el material tendría que desplegarse en su contenedor original ocupando varias estaciones de trabajo, complicando las operaciones asignadas a otro operario e incrementando caminatas.

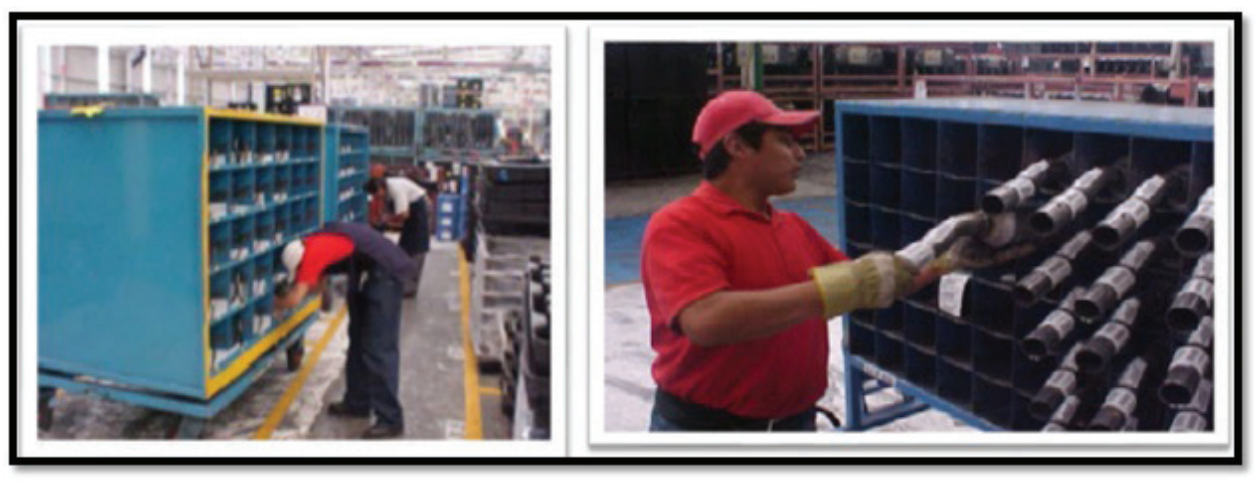

Figura 5. Operadores de línea

Elaboración propia

Después de utilizar al proveedor y modificar los carros secuenciadores, se logró un $85 \%$ de mejora en ciclos al enviar 80 secuencias por turno, en 20 ciclos directos al punto de uso en línea, reduciendo más del $50 \%$; considerando, entonces, que el costo del minuto de paro es de 127,50 dólares, se logró un ahorro de 24225 dólares, pasando de 47175 dólares a 22950 dólares al final del proyecto. La figura 6 muestra la reducción de los paros de línea por turno en donde se aplicó la mejora. 


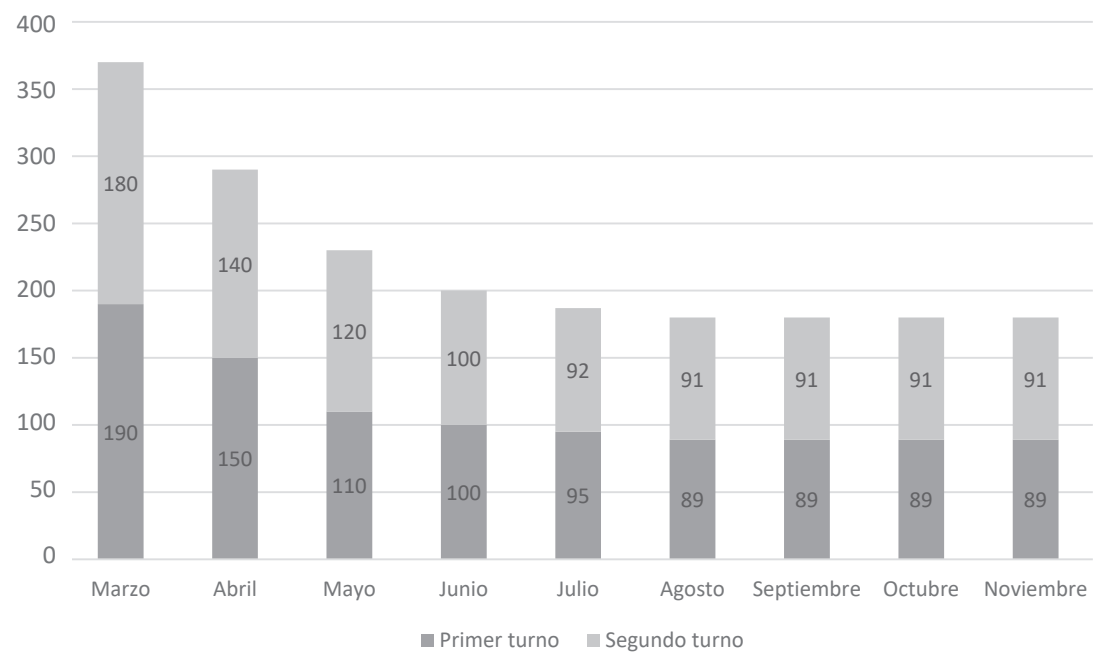

Figura 6. Disminución en paros de línea

Elaboración propia

\section{DISCUSIÓN Y CONCLUSIONES}

En la industria automotriz, para ser competitivo y estar a la vanguardia, se requiere contar con plantas de ensamble y manufactura flexible que impliquen la fabricación de dos o más modelos en una misma línea de producción. En este enfoque de manufactura, también llamada de grupos tecnológicos, ha sido ampliamente aprobada porque permite lograr una producción económica de una gran variedad de productos, mediante la efectiva integración de la administración y la planeación de la capacidad y de la producción.

Hay muchos estudios que demuestran la importancia que tiene para los sistemas productivos, la implementación de herramientas de manufactura esbelta. Según Jiménez-García et al. (2016) existe un programa de suministro de materiales para una secuencia de producción, el cual se implementó dentro de las primeras 3 horas del primer turno de trabajo, y logró reducir las pérdidas debido a la falta de suministros de material de $1,6 \%$ a $0 \%$, donde 1,6 \% (datos proporcionados por el ERP de la organización), representa el porcentaje de espera por falta de suministro de materiales en un turno de trabajo.

Martínez Sánchez et al. (2016) concluyen que la aplicación de lean manufacturing, con herramientas como el mapeo de procesos y el diagrama de recorrido, facilitaron la identificación de procesos y distancias a optimizar para la disminución en los tiempos de atención de pacientes, logrando mejoras en los tiempos de atención de hasta el 56 \%. 
Figueredo Lugo (2015) demuestra cómo la implementación de la filosofía de manufactura esbelta, en un proceso de producción de concreto premezclado, logró aumentar su rendimiento en $0,32 \%$, en comparación con la medición inicial, obteniendo con esto una mejora en el indicador OEE de 1,20\%, situándose en $65,29 \%$.

Este estudio muestra todo lo que se debe considerar logísticamente al referirnos, únicamente, al suministro de materiales y herramientas de manufactura esbelta para lograr que una planta ensambladora de autos pueda incrementar su eficiencia con secuencias de materiales, además, se logran grandes beneficios intangibles como una producción más flexible, estaciones de trabajo ergonómicas, mejoras en la seguridad y relaciones más estrechas con los proveedores. En resumen, con la elaboración de este trabajo se logró un ahorro hasta del 50 \% en la línea de producción al recorrer solo 800 de los 1600 metros que se tenían antes de la implementación de las herramientas de manufactura esbelta, de igual modo, se redujeron el $85 \%$ de los ciclos; actualmente, con solo 20 de ellos, se ganó un ahorro importante de 383751 dólares al entregar las partes del almacén, directamente, a la línea en el momento preciso en que se requiere, evitando así los paros de línea.

Debido a la naturaleza de este trabajo, a sus implicaciones y a la complejidad que encierra la operación de secuenciado de partes en una planta ensambladora de automóviles, es importante hacer mención que sobre algunos beneficios su resultado se verá reflejado a mediano plazo, ya que no debemos perder de vista que este es un trabajo planeado así. Es importante mencionar que los resultados de la secuenciación, actualmente, siguen siendo evaluados por la empresa con efectos satisfactorios dando la pauta para continuar implementando más secuencias.

\section{REFERENCIAS}

Acevedo Suárez, J. A., Urquiaga Rodríguez, A. J., y Gómez Acosta, M. (2001). Gestión de la cadena de suministro. La Habana: Centro de Estudio Tecnología de Avanzada (CETA) y Laboratorio de Logística y Gestión de la Producción (Logespro).

Anderson, D., y Bozheva, T. (2019). Kanban maturity model: desarrollando la agilidad organizativa y de negocio en empresas industriales. DYNA Management, 7(1). http://dx.doi.org/10.6036/MN8805

Arango Serna, M. D., Campuzano Zapata, L. F., y Zapata Cortes, J. A. (2015). Mejoramiento de procesos de manufactura utilizando Kanban. Revista Ingenierías Universidad de Medellín, 14(27), 221-233.

Bravo, J. J., Orejuela, J. P., y Menduiña, Z. (2012). Aproximación a la medición del impacto del backorder en sistemas de manufactura flexible. Revista Facultad de Ingeniería Universidad de Antioquia, 65, 99-111. 
Chase, R. B., Jacobs, F. R., y Aquilano, N. J. (2009). Administración de operaciones. Producción y cadena de suministros. México D. F.: McGraw-Hill.

Figueredo Lugo, F. J. (2015). Aplicación de la filosofía lean manufacturing en un proceso de producción de concreto. Ingeniería Industrial. Actualidad y Nuevas Tendencias, 4(15), 7-24.

Franco, J. M., Jimenez-García, J. A., y Ruelas-Santoyo, E. A. (2019). Minimization of the time for the materials supply through a mixed integer linear programming model. Dyna Management, 7(1). doi: http://dx.doi.org/10.6036/MN9037

Jiménez-García, J. A., Mendiola-García, J. Y., Medina-Flores, J. M., Mezura-Montes, E., y Vázquez-López, J. A. (2016). Reducing losses due to lack of supply in a manufacturing company using a mixed-integer linear programming model. International Journal of Combinatorial Optimization Problems and Informatics, 7(2), 3-12.

Lijalem, M. (2020). Productivity improvement through lean manufacturing tools in Ethiopian garment manufacturing company. Materials Today: Proceedings, 37(2), 1432-1436. https://doi.org/10.1016/j.matpr.2020.06.599

Martínez Sánchez, P., Martínez Flores, J., Nuño de la Parra, P., y Cavazos Arroyo, J. (2016). Mejora en el tiempo de atención al paciente en una unidad de urgencias ginecoobstétricas mediante la aplicación de lean manufacturing. Revista Lasallista de Investigación, 13(2), 46-56.

Oropesa-Vento, M., García-Alcaraz, J. L., Rivera, L., y Manotas, D. F. (2015). Effects of management commitment and organization of work teams on the benefits of Kaizen: Planning stage. Dyna, 82(191), 76-84.

Rodenes Adam, M., Moncaleano Rodríguez, G. I., y Martínez Aparisi, A. (2009). Importancia del outsourcing como apoyo de los servicios a la industria. Economía Industrial, $374,65-73$.

Rodríguez León, J., Quiroga Méndez, J. E., y Ortiz Pimiento, N. R. (2013). Performance comparison between a classic particle swarm optimization and a genetic algorithm in manufacturing cell design. Dyna, 80(178), 29-36.

Vargas-Hernández, J. G., Jiménez Castillo, M. T., y Muratalla-Bautista, G. (2018). Sistemas de producción competitivos mediante la implementación de la herramienta lean manufacturing. Ciencias Administrativas, 6(11), 81-95. https://doi. org/10.24215/23143738e020 